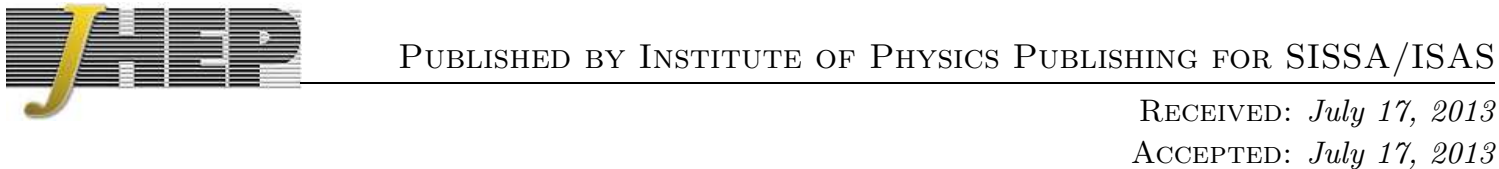

ACCEPTED: July 17, 2013

\title{
Lepton asymmetries and the growth of cosmological seed magnetic fields
}

\author{
V.B. Semikoz* \\ J. W. F. Valle \\ AHEP Group, Institut de Física Corpuscular - C.S.I.C./Universitat de València \\ Edificio Institutos de Paterna, Apt 22085, E-46071 Valencia, Spain. E-mail: \\ semikoz@ific.uv.es, valle@ific.uv.es
}

ABSTRACT: Primordial cosmological hypermagnetic fields polarize the early Universe plasma prior to the electroweak phase transition (EWPT). As a result of the long range parity violating gauge interaction present in the Standard Model their magnitude gets amplified, opening a new perturbative way of seeding the primordial Maxwellian magnetic field at EWPT.

KEYWords: Leptons, Neutrino interactions, Magnetohydrodynamics.

*Pushkov Institute of Terrestrial Magnetism, Ionosphere and Radiowave Propagation of the Russian Academy of Sciences, IZMIRAN, Troitsk, Moscow region, 142190, Russia. 
The electroweak phase transition (EWPT) has long been considered as a playing an important role in the generation of primordial magnetic fields [1, 2, 3]. Here we suggest that the seed fields accounting for the observed intergalactic magnetic fields arise, say, as tiny fluctuations $\sim 10^{-21}$ Gauss, associated to some early phase transition prior to the EWPT. The interplay between the resulting polarization effects of the early Universe plasma and the long range parity violating gauge interaction present in the Standard Model subsequently amplifies the seed field till an epoch close to the EWPT time, after which its evolution is described by standard perturbative physics, free from uncertainties from the EWPT epoch.

It is well-known that, at the high-temperature symmetric phase of the Standard Model (SM) all gauge bosons acquire a "magnetic" mass gap $\sim g^{2} T$, except for the Abelian gauge field associated to weak hypercharge. Collective plasma effects also modify the dispersion relations of the quarks and leptons at finite temperature giving them effective "chirallyinvariant" masses determined by their gauge group quadratic Casimirs [4].

While long-range magnetic-like fields associated to non-Abelian gauge forces do not exist, the Abelian hypercharge magnetic fields are never screened and can survive in the plasma for infinitely long times. The nonconservation of the lepton charge due to the Abelian anomaly associated with the hypercharge field $Y_{\mu}$ has been suggested to play a role in generating the observed the baryon asymmetry of the Universe [5].

Here we stress the importance of polarization effects in the early Universe plasma associated to the parity violating weak hypercharge interactions well before the EWPT. We show how, in the presence of a lepton asymmetry expected to arise in the leptogenesis scenario [6], a pre-existing field $B_{0}^{Y}$, for simplicity assumed to be a large-scale field, will undergo spectacular growth prior to the EWPT. Below the critical EWPT temperature the hypermagnetic fields are converted into standard Maxwellian magnetic fields. Thus the amplification mechanism can seed the ordinary magnetic field, helping to account for the observed magnitude of intergalactic magnetic fields [7].

Consider the equations of motion for the hypercharge $Y_{\mu}$-field in the hot plasma and in the presence of a pre-existing large-scale hypermagnetic field $\mathbf{B}_{0}^{Y}$, regular on scales smaller than the horizon size at $T_{0}>T \gg T_{E W P T}$. We assume that this primordial field has a small amplitude $g^{\prime} B_{0}^{Y} \ll T^{2}$. For simplicity, we neglect the Abelian anomaly and assume flat Minkowski space. The $U(1)_{Y}$ interaction Lagrangian in the SM with the one Higgs doublet $\varphi^{T}=\left(\phi^{(+)} \phi^{(0)}\right)$ is given as,

$$
\begin{aligned}
& \mathcal{L}_{i n t}=\sum_{\ell} \frac{g^{\prime} Y^{\mu}}{2}\left[-\bar{\nu}_{\ell L} \gamma_{\mu} \nu_{\ell L}-\bar{\ell}_{L} \gamma_{\mu} \ell_{L}-2 \bar{\ell}_{R} \gamma_{\mu} \ell_{R}\right]+ \\
& +\sum_{i}^{N} \frac{g^{\prime} Y^{\mu}}{2}\left[\frac{1}{3} \bar{U}_{i L} \gamma_{\mu} U_{i L}+\frac{1}{3} \bar{D}_{i L} \gamma_{\mu} D_{i L}+\frac{4}{3} \bar{U}_{i R} \gamma_{\mu} U_{i R}-\frac{2}{3} \bar{D}_{i R} \gamma_{\mu} D_{i R}\right]+ \\
& +i \frac{g^{\prime} Y^{\mu}}{2}\left[\varphi^{+} D_{\mu} \varphi-\left(D_{\mu} \varphi^{+}\right) \varphi\right] .
\end{aligned}
$$

For the assumed external seed hypermagnetic field $\mathbf{B}_{0}^{Y}=\nabla \times \mathbf{Y}^{(0)}=\left(0,0, B_{0}^{Y}\right)$, this leads to the following Dirac equations for massless charged leptons $(\ell=e, \mu, \tau)$, neutrinos and 
quarks $\left(U_{i}=u, c, t, D_{i}=d, s, b\right)$

$$
\left[\hat{p}-f^{(a)}\left(g^{\prime}\right) \hat{Y}^{(0)}\right] \Psi^{(a)}=0,
$$

where $a=\ell_{L}, \ell_{R}, \nu_{\ell}, U_{L}, D_{L}, U_{R}, D_{R}$ and $f^{(a)}\left(g^{\prime}\right)$ denote the corresponding SM couplings: $f_{R}\left(g^{\prime}\right)=-g^{\prime}$ for right-handed charged leptons, $f_{L}\left(g^{\prime}\right)=-g^{\prime} / 2$ for left-handed charged leptons and neutrinos, $f_{L}^{(U, D)}\left(g^{\prime}\right)=g^{\prime} / 6$ and $f_{R}^{(U)}\left(g^{\prime}\right)=2 g^{\prime} / 3, f_{R}^{(D)}\left(g^{\prime}\right)=-g^{\prime} / 3$ for lefthanded quarks and right-handed quarks respectively.

The resulting Landau spectrum in the mean hypercharge magnetic field takes the form $\varepsilon\left(p_{z}, n, \lambda\right)=\sqrt{p_{z}^{2}+\left|f_{L, R}\left(g^{\prime}\right)\right| B_{0}^{Y}(2 n+1 \mp \lambda)}$, where the upper sign applies to particles and the lower one to antiparticles, irrespective of their chiralities, $\lambda= \pm 1$. Within the small-field approximation $g^{\prime} B_{0}^{Y} \ll T^{2}$ we have

$$
\varepsilon(p, \lambda)=p+\left|f_{L, R}\left(g^{\prime}\right)\right| B_{0}^{Y} \lambda / 2 p
$$

with $p=\sqrt{p_{z}^{2}+p_{\perp}^{2}}$ and $p_{\perp}^{2}=\left|f_{L, R}\left(g^{\prime}\right)\right| B_{0}^{Y}(2 n+1)$. Note that such paramagnetic term in Eq. (2) coming from the spin of fermions is absent for Higgs bosons.

The above spectrum leads to the unique definition of the equilibrium distribution functions in the multi-particle approach. Up to the Abelian anomaly lepton (and quark) numbers are conserved through the continuity equations $\partial j_{\mu}^{(a)} / \partial x_{\mu}=0$ for $a=\ell_{L}, \ell_{R}, \nu_{\ell}, U_{L, R}, D_{L, R}$. Therefore we may define chemical potentials $\mu^{(a)}$ for the corresponding equilibrium distribution functions. From Eq. (2) we easily find the equilibrium one-particle density matrix for massless particles

$$
f_{\lambda^{\prime} \lambda}^{(a, \bar{a})}=\frac{\delta_{\lambda^{\prime} \lambda}}{\exp \left[\left(\varepsilon\left(p_{z}, n, \lambda\right) \mp \mu_{a}\right) / T\right]+1}
$$

which is approximated in the quasi-classical limit, by

$$
f_{\lambda^{\prime} \lambda}^{(a, \bar{a})} \simeq \frac{\delta_{\lambda^{\prime} \lambda}}{2} f_{0}^{(a, \bar{a})}(p)+\frac{\sigma_{\lambda^{\prime} \lambda}^{j}}{2} S_{0}^{(a, \bar{a}) j}(p),
$$

with the lower sign for chemical potentials corresponding to antiparticles. Here $f^{(a, \bar{a})}(p)=$ $\left[e^{\left(p \mp \mu_{a}\right) / T}+1\right]^{-1}$ are the Fermi distributions of particles (antiparticles) which for $\mu_{a} / T \ll 1$ correspond to the Lorentz-invariant densities $n^{(a, \bar{a})}$,

$$
\begin{aligned}
& n^{(a, \bar{a})}=\int \frac{d^{3} p}{(2 \pi)^{3}} \frac{1}{\exp \left(\left[p \mp \mu_{a}\right] / T\right)+1} \approx \\
& \approx n_{e q}\left[1 \pm \frac{\pi^{2}}{9 \zeta(3)}\left(\frac{\mu_{a}}{T}\right)+O\left(\left(\frac{\mu_{a}}{T}\right)^{2}\right)\right] .
\end{aligned}
$$

Here $n_{e q}=3 \zeta(3) T^{3} / 4 \pi^{2}$ is the equilibrium lepton density when $\mu_{a}=0 ; \zeta(3) \approx 1.202$ is the Riemann function value. The second term in Eq. (3) includes Pauli matrices multiplied by the mean spin vector,

$$
\mathbf{S}_{0}^{(a, \bar{a})}(p)=-\frac{\left|f_{a}\left(g^{\prime}\right)\right| \mathbf{B}_{0}^{Y}}{2 p} \frac{\mathrm{d} f_{0}^{(a, \bar{a})}(p)}{\mathrm{d} p}=\hat{\mathbf{b}}_{0} S_{0}^{(a, \bar{a})}(p)
$$


which is the equilibrium spin distribution function. Here $\hat{\mathbf{b}}_{0}=\mathbf{B}_{0}^{Y} / B_{0}^{Y}$ is the unit vector along the mean hypermagnetic field. Notice that equilibrium spin distribution values $S_{0}^{(a, \bar{a})}(p)$ define the densities of fermions populating the main Landau level, $n=0$ :

$$
\begin{aligned}
& n_{0}^{(a, \bar{a})}=\int \frac{d^{3} p}{(2 \pi)^{3}} S_{0}^{(a, \bar{a})}(p) \\
& =\frac{\left|f_{a}\left(g^{\prime}\right)\right| B_{0}^{Y} T \ln 2}{4 \pi^{2}}\left[1 \pm \frac{\mu_{a}}{2 T \ln 2}+O\left(\left(\frac{\mu_{a}}{T}\right)^{2}\right)\right] .
\end{aligned}
$$

Note also that the subindex $\lambda$ in the density matrix corresponds to the spin projection on the hypermagnetic field, $\left(\sigma_{z}\right)_{\lambda^{\prime} \lambda}=\lambda \delta_{\lambda^{\prime} \lambda}$. Together with chirality $\gamma_{5} \Psi_{e_{R, L}}= \pm \Psi_{e_{R, L}}$ it is a good quantum number since $\left[\gamma_{5}, \Sigma_{z}\right]=0$.

Note that the density asymmetry for massless fermions coming from Eq. (4) , $n^{(a)}-$ $n^{(\bar{a})}=\mu_{a} T^{2} / 6$, is one half that of the Higgs bosons $\left(\mu_{a} T^{2} / 3\right)$. Taking also into account the equilibrium conditions for the chemical potentials of the plasma components (using plasma neutrality $\langle Q\rangle=0,\left\langle Q_{3}\right\rangle=\mu_{W}=0$ [9]) and normalizing the hypercharge $Y$ on $T^{2} / 6$ where $Y$ directly reads from the Lagrangian Eq. (11) as ${ }^{1}$,

$$
\begin{aligned}
& Y=-\sum_{l}\left(\mu_{\nu_{l} L}+\mu_{L}^{l}\right)-2 \sum_{l} \mu_{R}^{l}+N\left[\mu_{u L}+\mu_{d L}+4 \mu_{u R}-2 \mu_{d R}\right]+2\left(\mu_{+}+\mu_{0}\right)= \\
& =2\left(Q-Q_{3}\right)=2\left[-2 \sum_{l} \mu_{L}^{l}+6 \mu_{u L}+14 \mu_{0}\right]=0,
\end{aligned}
$$

one finds the chemical potential of the neutral Higgs boson $\mu_{0}$,

$$
\mu_{0}=\frac{\sum_{l} \mu_{L}^{l}-3 \mu_{u L}}{7}
$$

Using the sphaleron equilibrium condition valid above EWPT,

$$
\sum_{l} \mu_{L}^{l}=-9 \mu_{u L}
$$

and the expressions for the baryon and lepton numbers in terms of chemical potentials (given in Eq. (8) in Ref. [9]) one can see that $\mu_{0}$ vanishes only in the exceptional case $B=L=0$ (see, e.g. in [10]).

Note that in the first line of Eq. (7) we have used $\mu_{+}=-\mu_{-}=\mu_{0}$. This follows from the equilibrium relation $\mu_{W}=\mu_{-}+\mu_{0}$ (implied by conversions $W^{-} \leftrightarrow \phi^{-}+\phi^{0}$ ) and the neutrality condition for the isospin component $Q_{3} \sim \mu_{W}=0$. We also took into account the common color factor of quarks and assumed that quark asymmetries are degenerate in flavor for all left-handed up-quark fields and all right-handed down-quark fields respectively (note that we do not assume such degeneracy for charged leptons and neutrinos).

One can easily write down Maxwell-like equations of motion for the Abelian $Y_{\mu}$-field in the equilibrium plasma. These involve thermal averages of the various four-current fermion densities, e. g. for leptons $\left\langle\bar{\ell}_{a} \gamma_{\mu} \ell_{a}>\right.$, given as differences of Wigner distribution functions

\footnotetext{
${ }^{1}$ The same result follows from the definition of hypercharge $Y$ in Eq. (8) in paper [8].
} 
for particles and antiparticles, $\delta f_{\lambda^{\prime} \lambda}^{(a)}(\mathbf{p}, \mathbf{x}, t)=f_{\lambda^{\prime} \lambda}^{(a)}(\mathbf{p}, \mathbf{x}, t)-f_{\lambda^{\prime} \lambda}^{(\bar{a})}(\mathbf{p}, \mathbf{x}, t)$. Substituting the general distribution function,

$$
f_{\lambda^{\prime} \lambda}^{(a)}(\mathbf{p}, \mathbf{x}, t)=\frac{\delta_{\lambda^{\prime} \lambda}}{2} f^{(a)}(\mathbf{p}, \mathbf{x}, t)+\frac{\left(\sigma_{i}\right)_{\lambda^{\prime} \lambda}}{2} S_{i}^{(a)}(\mathbf{p}, \mathbf{x}, t)
$$

and summing over spin variables $\lambda, \lambda^{\prime}$ one can recast the Poisson and Maxwell-like equations as

$$
\begin{aligned}
& \nabla \cdot \mathbf{B}_{Y}=0, \quad \nabla \cdot \mathbf{E}_{Y}=4 \pi\left[J_{0}^{Y}(\mathbf{x}, t)+J_{05}^{Y}(\mathbf{x}, t)\right], \\
& \frac{\partial \mathbf{B}_{Y}}{\partial t}=-\nabla \times \mathbf{E}_{Y}, \\
& -\frac{\partial \mathbf{E}_{Y}}{\partial t}+\nabla \times \mathbf{B}_{Y}=4 \pi\left[\mathbf{J}^{Y}(\mathbf{x}, t)+\mathbf{J}_{5}^{Y}(\mathbf{x}, t)\right],
\end{aligned}
$$

These describe the equilibrium plasma at $T \gg T_{E W P T}$ and differ from the familiar Maxwell equations by the presence of the pseudovector current $J_{\mu 5}^{Y}$ associated to the parity violating $Y_{\mu}$ interactions.

The vector current is a sum $J_{\mu}^{Y}=\sum_{\ell} J_{\ell \mu}^{Y}+3 N J_{(q) \mu}^{Y}+J_{(\varphi) \mu}^{Y}$ where each term is given in terms of differences of current asymmetries

$$
\delta j_{\mu}^{(a)}=j_{\mu}^{(a)}-j_{\mu}^{(\bar{a})}=\int \frac{d^{3} p}{(2 \pi)^{3}} \frac{p_{\mu}}{p} \delta f^{(a)}(\mathbf{p}, \mathbf{x}, t)
$$

in the form following from the interaction Lagrangian (11),

$$
\begin{aligned}
& J_{\ell \mu}^{Y}(\mathbf{x}, t)=-\frac{g^{\prime}}{4}\left[2 \delta j_{\mu}^{\ell_{R}}(\mathbf{x}, t)+\delta j_{\mu}^{\ell_{L}}(\mathbf{x}, t)+\delta j_{\mu}^{\nu_{\ell L}}(\mathbf{x}, t)\right], \\
& J_{(q) \mu}^{Y}(\mathbf{x}, t)=\frac{g^{\prime}}{12}\left[\left(\delta j_{\mu}^{(u L)}(\mathbf{x}, t)+\delta j_{\mu}^{(d L)}(\mathbf{x}, t)\right)+4 \delta j_{\mu}^{(u R)}(\mathbf{x}, t)-2 \delta j_{\mu}^{(d R)}(\mathbf{x}, t)\right], \\
& J_{(\varphi) \mu}^{Y}(\mathbf{x}, t)=\frac{g^{\prime}}{2} \delta j_{\mu}^{(\varphi)}(\mathbf{x}, t),
\end{aligned}
$$

involving partial current asymmetries given as

$$
\delta j_{\mu}^{(a)}=\left\{\left(n^{(a)}-n^{(\bar{a})}\right) \gamma ; \quad n_{e q} \gamma\left(\mathbf{V}^{(a)}-\mathbf{V}^{(\bar{a})}\right)\right\},
$$

where $n^{(a)} \equiv n^{(a)}(\mathbf{x}, t)=j_{\mu}^{(a)}(\mathbf{x}, t) u^{\mu}(\mathbf{x}, t)$ are the densities defined in Eq. (41) which coincide with those calculated in the medium rest frame, $\mathbf{V}=0, \gamma=\left(1-V^{2}\right)^{-1 / 2}=$ 1. Summing the individual contributions in Eq. (12) one obtains the hypercharge vector current density $J_{\mu}^{Y}$ as

$$
\begin{aligned}
& J_{0}^{Y}=-\gamma n_{e q}\left(\frac{2 \pi^{2}}{9 \zeta(3)}\right)\left(\frac{g^{\prime}}{4 T}\right)\left[-2 \sum_{l} \mu_{L}^{l}+6 \mu_{u L}+14 \mu_{0}\right], \\
& \mathbf{J}^{Y}=\sum_{a} \frac{f^{(a)}\left(g^{\prime}\right)}{2} \gamma n_{e q}\left[\mathbf{V}^{(a)}-\mathbf{V}^{(\bar{a})}\right] .
\end{aligned}
$$


As seen from Eq. (7) hypercharge neutrality of the plasma $\langle Y\rangle=0$ implies the vanishing the component $J_{0}^{Y}=0$ at large scales exceeding the Debye radius, $r \gg r_{D}$, resulting in the relation between chemical potentials Eq. (8).

We now turn to the axial current. Using Eq. (10) one can express the axial vector current $J_{\mu 5}^{Y}(\mathbf{x}, t)$ in terms of differences of spin distribution functions entering the axial fourvectors $\delta A_{\mu}^{(a)}(\mathbf{p}, \mathbf{x}, t)=A_{\mu}^{(a)}(\mathbf{p}, \mathbf{x}, t)-A_{\mu}^{(\bar{a})}(\mathbf{p}, \mathbf{x}, t)$ with $A_{\mu}^{(a)}(\mathbf{p}, \mathbf{x}, t)=\left\{\mathbf{p} \cdot \mathbf{S}^{(a)}(\mathbf{p}, \mathbf{x}, t)\right) ;(\mathbf{p}$. $\left.\left.\mathbf{S}^{(a)}(\mathbf{p}, \mathbf{x}, t)\right) \mathbf{p} / p\right\}^{2}$.

The total pseudovector current $J_{\mu 5}^{Y}=\sum_{l} J_{l \mu 5}^{Y}(\mathbf{x}, t)+3 N J_{q \mu 5}^{Y}$ is given in terms of partial contributions of leptons and quarks:

$$
\begin{aligned}
& J_{\ell \mu 5}^{Y}(\mathbf{x}, t)=-\frac{g^{\prime}}{2} \int \frac{d^{3} p}{p(2 \pi)^{3}} \delta A_{\mu}^{(\ell R)}(\mathbf{p}, \mathbf{x}, t)+\frac{g^{\prime}}{4} \int \frac{d^{3} p}{p(2 \pi)^{3}} \delta A_{\mu}^{(\ell L)}(\mathbf{p}, \mathbf{x}, t)+ \\
& +\frac{g^{\prime}}{4} \int \frac{d^{3} p}{p(2 \pi)^{3}} \delta A_{\mu}^{(\nu \ell)}(\mathbf{p}, \mathbf{x}, t), \\
& J_{q \mu 5}^{Y}=-\frac{g^{\prime}}{12} \int \frac{d^{3} p}{p(2 \pi)^{3}} \delta A_{\mu}^{(u L)}(\mathbf{p}, \mathbf{x}, t)-\frac{g^{\prime}}{12} \int \frac{d^{3} p}{p(2 \pi)^{3}} \delta A_{\mu}^{(d L)}(\mathbf{p}, \mathbf{x}, t)+ \\
& +\frac{g^{\prime}}{3} \int \frac{d^{3} p}{p(2 \pi)^{3}} \delta A_{\mu}^{(u R)}(\mathbf{p}, \mathbf{x}, t)-\frac{g^{\prime}}{6} \int \frac{d^{3} p}{p(2 \pi)^{3}} \delta A_{\mu}^{(d R)}(\mathbf{p}, \mathbf{x}, t) .
\end{aligned}
$$

Substituting the equilibrium spin distribution Eq. (5) into Eq. (15) we immediately get $J_{05}=0$ in the rest frame of the medium as a whole, $\mathbf{V}=0, \gamma=1$. Thus, under the hypercharge neutrality condition in Eq. (7), one has that $J_{0}^{Y}=J_{05}^{Y}=0$, so that the Poisson equation takes the standard form, $\nabla \cdot \mathbf{E}_{Y}=0$, in agreement with Ref. [5]. (Note, however, that small scale fluctuations described by non-equilibrium distribution functions $\delta f^{(a)}(\mathbf{p}, \mathbf{x}, t), \delta \mathbf{S}^{(a)}(\mathbf{p}, \mathbf{x}, t)$ could lead to $\left.\delta J_{0}^{Y} \neq 0, \delta J_{05} \neq 0\right)$.

In contrast, each pseudovector 3 -current $\mathbf{J}_{\ell 5}^{Y}$ or $\mathbf{J}_{q 5}^{Y}$ in Eq. (15) is nonzero even in equilibrium,

$$
\begin{aligned}
& \left(\mathbf{J}_{\ell 5}^{Y}\right)_{e q}=-\frac{g^{\prime}}{2}\left[\mathbf{j}_{5}^{\ell R}-\mathbf{j}_{5}^{\bar{\ell} R}\right]+\frac{g^{\prime}}{4}\left[\mathbf{j}_{5}^{\ell L}-\mathbf{j}_{5}^{\bar{\ell} L}\right]+\frac{g^{\prime}}{4}\left[\mathbf{j}_{5}^{\nu_{\ell}}-\mathbf{j}_{5}^{\bar{\nu}_{\ell}}\right], \\
& \left(\mathbf{J}_{q 5}^{Y}\right)_{e q}=-\frac{g^{\prime}}{12}\left[\mathbf{j}_{5}^{u L}-\mathbf{j}_{5}^{\bar{u} L}\right]-\frac{g^{\prime}}{12}\left[\mathbf{j}_{5}^{d L}-\mathbf{j}_{5}^{\bar{d} L}\right]+\frac{g^{\prime}}{3}\left[\mathbf{j}_{5}^{u R}-\mathbf{j}_{5}^{\bar{u} R}\right]-\frac{g^{\prime}}{6}\left[\mathbf{j}_{5}^{d R}-\mathbf{j}_{5}^{\bar{d} R}\right] .
\end{aligned}
$$

Substituting the equilibrium spin distributions in Eq. (5) and using again the hypercharge neutrality condition and the sphaleron equilibrium condition Eq. (9) we get, after summing over leptons $\sum_{\ell}$ and quarks,

$$
\left(\mathbf{J}_{5}^{Y}\right)_{e q}=\frac{g^{\prime 2}}{96 \pi^{2}}\left[-2 \sum_{l} \mu_{L}^{l}+10 \mu_{u L}+32 \mu_{0}\right] \mathbf{B}_{0}^{Y}=\frac{47}{1512 \pi^{2}} g^{\prime 2} \mu_{\nu} \mathbf{B}_{0}^{Y},
$$

which leaves then only one independent lepton asymmetry which we take as that of neutrinos, $\sum_{l} \mu_{L}^{l}=\sum_{l} \mu_{\nu_{l L}}=\mu_{\nu}$. Notice that we have used here Eqs. (8), (9) for the chemical potentials $\mu_{0}$ and $\mu_{u L}$.

\footnotetext{
${ }^{2}$ This is the massless limit of the four-vector spin distribution that generalizes the Pauli-Lubanski spin vector in the multi-particle approach 13], $A_{\mu}^{(a)}(\mathbf{p}, \mathbf{x}, t)=m_{a} \times a_{\mu}=\lim _{m_{a} \rightarrow 0} m_{a} \times\left[\mathbf{p S}^{(a)} / m_{a} ; \mathbf{S}^{(a)}+\right.$ $\left.\left(\mathbf{p} \mathbf{S}^{(a)}\right) \mathbf{p} / m_{a}\left(\varepsilon_{p}+m_{a}\right)\right]$, where $\mathbf{S}^{(a)}=\mathbf{S}^{(a)}(\mathbf{p}, \mathbf{x}, t)$. Note that the standard Lorenz-invariant normalization $a_{\mu} a^{\mu}=-\mathbf{S}^{2} \neq 0$ is replaced by $A_{\mu}^{(a)} A^{\mu(a)}=0$ in the massless fermion case.
} 
Thus, the system of Magneto-Hydro-Dynamics (MHD) equations for hyperelectromagnetic fields in Eq. (11) finally gets the form:

$$
\begin{aligned}
& \nabla \cdot \mathbf{B}_{Y}=0, \quad \nabla \cdot \mathbf{E}_{Y}=0, \\
& \frac{\partial \mathbf{B}_{Y}}{\partial t}=-\nabla \times \mathbf{E}_{Y}, \\
& -\frac{\partial \mathbf{E}_{Y}}{\partial t}+\nabla \times \mathbf{B}_{Y}=4 \pi \mathbf{J}^{Y}+\frac{47}{378} \times \frac{g^{\prime 2} \mu_{\nu}}{\pi} \mathbf{B}_{0}^{Y} .
\end{aligned}
$$

Averaging the total field $\mathbf{B}_{Y}=\mathbf{B}_{0}^{Y}+\mathbf{b}_{Y}(\mathbf{x}, t)$ over random small-scales, $\left\langle\mathbf{b}_{Y}\right\rangle=0$, we can rewrite Eq. (18) as an MHD system for mean hypermagnetic fields $\mathbf{B}_{Y}=\mathbf{B}_{0}^{Y}$ completed by the Ohm equation. In the rest frame $\mathbf{V}=0$ of the isotropic early Universe plasma we are considering the Ohm equation reduces to

$$
\mathbf{J}^{Y}=\sigma_{\text {cond }} \mathbf{E}_{Y} \text {. }
$$

One sees that the MHD equations derived here in the standard Weinberg-Salam model qualitatively coincide with what one obtains using the anomaly term in the interaction Lagrangian [2, 11].

By combining the Ohm law Eq. (19) and the last Maxwell-like equation in Eq. (18) then using $\partial \mathbf{B}_{Y} / \partial t=-\nabla \times \mathbf{E}_{Y}$, we can write the Faraday equation describing the so-called $\alpha^{2}$-dynamo [7] of hypermagnetic field as

$$
\frac{\partial \mathbf{B}_{Y}}{\partial t}=\nabla \times \alpha \mathbf{B}+\eta \nabla^{2} \mathbf{B}_{Y}
$$

where $\eta=\left(4 \pi \sigma_{\text {cond }}\right)^{-1}$ is the magnetic diffusion coefficient and we neglect, as usual in MHD, the displacement current $\partial \mathbf{E}_{Y} / \partial t$ and use the rest frame condition $\mathbf{V}=0$. The parameter $\alpha$ is the hypermagnetic helicity coefficient given as

$$
\alpha=\frac{47 g^{\prime 2} \mu_{\nu}}{1512 \pi^{2} \sigma_{\text {cond }}}
$$

and plays crucial role in the evolution of the hypermagnetic field. We can solve Eq. (20) through Fourier harmonics as $\mathbf{B}_{Y}(\mathbf{x}, t)=\int\left(d^{3} k /(2 \pi)^{3} \mathbf{B}_{\mathbf{Y}}(\mathbf{k}, t) e^{i \mathbf{k x}}\right.$ where $B_{Y}(k, t)$ is expressed as

$$
\left.B_{Y}(k, t)=B_{0}^{Y} \exp \left[\int_{t_{0}}^{t}\left[\alpha\left(t^{\prime}\right) k-\eta\left(t^{\prime}\right) k^{2}\right)\right] \mathrm{d} t^{\prime}\right] .
$$

For $0<k<\alpha / \eta$, or correspondingly correlation length scales $\eta / \alpha<\Lambda<\infty$ such field gets exponentially amplified, but differently for different scales $\Lambda$. E.g. for the Fourier mode $k=\alpha / 2 \eta$ (or $\Lambda \sim 2 \eta / \alpha$ ) one gets the maximum amplification $\gamma=\alpha k-\eta k^{2}=\alpha^{2} / 4 \eta$ [7, 12

$$
B_{Y}(t)=B_{0}^{Y} \exp \left[\int_{t_{0}}^{t} \frac{\alpha^{2}\left(t^{\prime}\right)}{4 \eta\left(t^{\prime}\right)} \mathrm{d} t^{\prime}\right]
$$

or

$$
B_{Y}(x)=B_{0}^{Y} \exp \left[32 \int_{x}^{x_{0}} \frac{\mathrm{d} x^{\prime}}{x^{\prime 2}}\left(\frac{\xi_{\nu}\left(x^{\prime}\right)}{0.001}\right)^{2}\right]
$$


where we introduced the new variable $x=T / T_{E W P T}$ and $B_{0}^{Y}$ is the assumed initial amplitude of the hypermagnetic field at $T_{0} \gg T_{E W P T}$. In the second equality we substituted $\eta=\left(4 \pi 137 T \cos ^{2} \theta_{W}\right)^{-1}$, the analogue of the magnetic diffusion coefficent taking into account the change from the standard QED fine structure constant $\left(e^{2} \sim 137^{-1}\right)$ to the analogous hypercharge one, with $e^{2} \rightarrow g^{\prime 2}=e^{2} / \cos ^{2} \theta_{W}$, where $\sin ^{2} \theta_{W}=0.23$ is the electroweak mixing parameter. We have also used the appropriate cosmological time-temperature relation $t=\left[3.84 \times 10^{21}(T / \mathrm{MeV})^{-2} / \sqrt{g^{*}}\right] \mathrm{MeV}^{-1}$ with the number of relativistic degrees of freedom $g^{*} \sim 100$. One sees that, even for small values of the lepton asymmetry $\xi_{\nu}$ one obtains a very strong amplification of the seed hypermagnetic field $B_{0}^{Y}$ at this scale.

Unfortunately, the unknown dependence of $\xi_{\nu}(x)$ for $x=T / T_{E W P T}>1$ prevents us from a reliable numerical estimate for the amplitude $B_{Y}(x)$. Moreover, since the evolution of $\xi_{\nu}$ depends on nonperturbative physics, it is not easy at this stage to confront it with the primordial nucleosynthesis bounds [14]. However, in order to survive against ohmic dissipation due to finite conductivity $\eta=\left(4 \pi \sigma_{\text {cond }}\right)^{-1}$ we should have $\Lambda>l_{\text {diff }}$, where $l_{d i f f}=\sqrt{\eta l_{H}}$ is the diffusion length. This leads to an upper bound on $\xi_{\nu}(x)=\mu_{\nu}(T) / T$,

$$
\frac{\xi_{\nu}(x)}{0.001}<A(\Lambda) \sqrt{x}, \quad x \geq 1
$$

which explicitly depends on the chosen scale $\Lambda$. Hence for the mode $\Lambda_{1}=2 \eta / \alpha$ one gets $A=0.23$ so that the amplification factor is $\sim 32$ as seen in Eq. (23), while for a larger scale, say $\Lambda_{2}=16 \eta / \alpha$, one gets $A=1.83$ with a reduced growth factor $\sim 7.4$ coming from the general solution in Eq. (22):

$$
B_{Y}(t)=B_{0}^{Y} \exp \left[\frac{15}{256} \int_{t_{0}}^{t} \frac{\alpha^{2}\left(t^{\prime}\right)}{\eta\left(t^{\prime}\right)} \mathrm{d} t^{\prime}\right]=B_{0}^{Y} \exp \left[7.4 \int_{x}^{x_{0}} \frac{\mathrm{d} x^{\prime}}{x^{\prime 2}}\left(\frac{\xi_{\nu}\left(x^{\prime}\right)}{0.001}\right)^{2}\right]
$$

In any case one can have very strong amplification even for larger scales for which the factor $A(\Lambda)$ is bigger.

In contrast to the mechanism suggested in Refs. [2] and [5] ours does not rely on the Chern-Simons anomaly term in the SM Lagrangian [11]. The presence of the anomaly acting at the later EWPT epoch, could play an important role in the subsequent evolution of the lepton asymmetry produced by the parity violating hypercharge interaction. However, by then the asymmetry has already induced the strong Maxwellian magnetic fields which no longer convert to leptons, as these carry no anomaly, their evolution being described by standard MHD equations. Here we do not study the EWPT conversion of hypercharge field to the Maxwellian magnetic field B. However we note that the seed value $B_{Y} \sim 0.3 T^{2}<$ $T_{E W P T}^{2} \sim 10^{24}$ Gauss can be easily reached through our Eqs. (23), (25). This provides a strongly first order EWPT that, in turn, allows to avoid the sphaleron constraint for baryogenesis within the Standard Model [15]. It is also important to note that our bound on the neutrino asymmetry in Eq. (24) provides the large scale of the mean hypermagnetic field $\Lambda \simeq L_{0}$ in Eq. (1) of ref. [15], so that bubble formation during EWPT takes place in the background of essentially constant field [15]. The subsequent evolution of the Maxwellian field proceeds through the inverse cascade [16]. 
Let us now comment on the physical interpretation of the new magnetic helicity term. The original seed field $B_{0}^{Y}$ polarizes the fermions and antifermions (including neutrinos) propagating along the field in the main Landau level, $n=0$. This polarization effect causes fermions and antifermions to move in opposite directions with a relative drift velocity proportional to the lepton asymmetry. The existence of a basic parity violating hypercharge interaction in the SM induces a new term in the hypermagnetic field in Eq. (20) $\nabla \times \alpha \mathbf{B}_{Y}$ which winds around the rectilinear pseudovector hypercharge current $\mathbf{J}_{5}$ parallel to $\mathbf{B}_{Y}$. This term amplifies the seed hypermagnetic field $B_{0}^{Y}$ according to Eqs. (22) and (23). It is interesting also to consider in detail the hypermagnetic helicity $H=\int \mathrm{d}^{3} x \mathbf{Y B}_{Y}$ and how it becomes the magnetic one below the EWPT [17.

In summary, while we are far from having a complete and fully quantitative picture for the origin of intergalactic magnetic fields, we think that the amplification mechanism described here could play an important role towards the goal of accounting for the observed intergalactic magnetic fields from first principles.

\section{Acknowledgments}

Work supported by MEC grant FPA2005-01269, by EC Contracts RTN MRTN-CT-2004503369 and ILIAS/N6 RII3-CT-2004-506222 and ACOMP07/270 of Generalitat Valenciana. V. B. S. thanks support from CSIC-RAS. We thank discussions with Dimitri Sokoloff.

\section{References}

[1] T. Vachaspati, Magnetic fields from cosmological phase transition, Phys. Lett. B265 (1991) 258.

[2] M. Joyce and M. E. Shaposhnikov, Primordial Magnetic Fields, Right electrons, and Abelian Anomaly, Phys. Rev. Lett. 79 (1997) 1193 [astro-ph/9703005].

[3] For reviews see D. Grasso and H. R. Rubinstein, Magnetic fields in the early Universe, Phys. Rept. 348 (2001) 163 [astro-ph/0009061]; M. Giovannini, The Magnetized Universe, Int. J. Mod. Phys. D 13 (2004) 391 [astro-ph/0312614].

[4] H. A. Weldon, Effective fermion masses of order $g T$ in high-temperature gauge theories with exact chiral invariance, Phys. Rev. D26 (1982) 2789.

[5] M. Giovannini and M. E. Shaposhnikov, Primordial hypermagnetic fields and the triangle anomaly, Phys. Rev. D57 (1998) 2186 [hep-ph/9710234].

[6] M. Fukugita and T. Yanagida, Baryogenesis Without Grand Unification, Phys. Lett. B 174 (1986) 45.

[7] I. B. Zeldovich, A. A. Ruzmaikin and D. D. Sokoloff, Magnetic fields in astrophysics (New York, Gordon and Breach Publishers), The Fluid Mechanics of Astrophysics and Geophysics. Volume 3, 1983, 381 p. Translation.

[8] S. Yu. Khlebnikov and M .E. Shaposhnikov, Melting of the Higgs Vacuum: Conserved Numbers at High Temperature, Phys. Lett. B387 (1996) 817 [hep-ph/9607386] 
[9] J. A. Harvey and M. S. Turner, Cosmological baryon and lepton number in the presence of electroweak fermion-number violation, Phys. Rev. D42 (1990) 3344.

[10] J.M. Cline, K. Kainulainen and K.A. Olive, Protecting the Primordial Baryon Asymmetry From Erasure by Sphalerons, Phys. Rev. D49 (1994) 6394.

[11] A. N. Redlich and L. C. R. Wijewardhana, Induced Chern-Simons terms at high temperatures and finite densities, Phys. Rev. Lett. 54 (1985) 970.

[12] V. B. Semikoz and D. D. Sokoloff, Large-Scale Magnetic Field Generation by $\alpha$-Effect Driven by Collective Neutrino-Plasma Interaction, Phys. Rev. Lett. 92 (2004) 131301 [astro-ph/0312567].

[13] V. B. Semikoz, Kinetics of a lepton plasma in the standard model ofelectroweak interactions, Physica 142A (1987) 157.

[14] A. D. Dolgov et al, Cosmological bounds on neutrino degeneracy improved by flavor oscillations, Nucl. Phys. B 632 (2002) 363 [hep-ph/0201287].

[15] P. Elmfors, K. Enqvist, K. Kainulainen, Strongly first order electroweak phase transition induced by primordial hypermagnetic field, Phys. Lett. B440 (1998) 269 [hep-ph/9806403].

[16] A. Brandenburg, K. Enqvist and P. Olesen, Large-scale magnetic fields from hydromagnetic turbulence in the very early universe, Phys. Rev. D 54 (1996) 1291 [astro-ph/9602031].

[17] V.B. Semikoz, D.D. Sokoloff, Magnetic helicity and cosmological magnetic field, Astronomy and Astrophysics, 433 (2005) L53 [astro-ph/0411496]. 